\title{
ON SIMPLE RUIN EXPRESSIONS IN DEPENDENT SPARRE ANDERSEN RISK MODELS
}

\author{
HANSJÖRG ALBRECHER, ${ }^{*}$ University of Lausanne \\ ONNO J. BOXMA, ${ }^{* *}$ Eindhoven University of Technology and EURANDOM \\ JEVGENIJS IVANOVS, ${ }^{* * * *}$ University of Lausanne
}

\begin{abstract}
In this note we provide a simple alternative probabilistic derivation of an explicit formula of Kwan and Yang (2007) for the probability of ruin in a risk model with a certain dependence between general claim interoccurrence times and subsequent claim sizes of conditionally exponential type. The approach puts the type of formula in a general context, illustrating the potential for similar simple ruin probability expressions in more general risk models with dependence.
\end{abstract}

Keywords: Sparre Andersen risk model; ruin probability; Markov additive process

2010 Mathematics Subject Classification: Primary 91B30; 97M30; 60K20

\section{Introduction}

Consider a renewal process $N_{t}$ with inter-epoch times $A_{k}$, and let

$$
Y_{t}=x+c t-\sum_{k=1}^{N_{t}} B_{k}
$$

be the surplus process of an insurance portfolio with initial capital $x \geq 0$, premium intensity $c \geq 0$, and claims $B_{k}$. The study of the ruin probability $\psi(x)=\mathbb{P}_{x}(T<\infty)$, with $T=$ $\inf \left\{t \geq 0: Y_{t}<0\right\}$, is a classical topic in risk theory. Whereas the usual assumption is that the independent and identically distributed sequences of random variables $\left(A_{k}\right)_{k \geq 1}$ and $\left(B_{k}\right)_{k \geq 1}$ are independent (in which case the model is referred to as the Sparre Andersen model), in recent years there has been increased interest in models with certain types of dependence. In that context, in [3] a model was considered in which $A_{k+1}$ depends on the previous claim size $B_{k}$ and some explicit expressions for the probability of ruin in this setting were derived. In [4] this analysis was extended to a semi-Markovian risk model (see also [2]). Due to a sample path duality (see, e.g. [7, Chapter III.2]), risk processes of that type have a counterpart in workload models of queueing theory, and a similar semi-Markovian structure was considered in [1] in a queueing context. In [5] it was proposed to assume that $\left(A_{k}, B_{k}\right)$ are independent and identically distributed pairs of positive random variables, but, for each $k, A_{k}$ and $B_{k}$ may be dependent. Under this assumption, the random walk structure of the process (when observed immediately

Received 19 December 2012; revision received 1 May 2013.

* Postal address: Department of Actuarial Science, Faculty of Business and Economics, University of Lausanne, UNIL-Dorigny, 1015 Lausanne, Switzerland.

** Postal address: Eindhoven University of Technology, PO Box 513, 5600 MB Eindhoven, The Netherlands. Email address: o.j.boxma@tue.nl

*** Supported by the Swiss National Science Foundation Project 200021-124635/1. 
after the claim occurrences only) is still preserved and allows for some explicit analysis (this model is also referred to as the dependent Sparre Andersen model; cf. [11]). In [8] it was shown that an explicit expression for $\psi(x)$ could be obtained for a particular type of dependence between $A_{k}$ and $B_{k}$. In [15], another explicit dependence structure in the framework of [5] was considered: if $A_{k}<a$ then $B_{k}$ is distributed according to $B^{(1)}$, otherwise according to $B^{(2)}$, where $a$ is a fixed threshold. It was shown in [15] that the ruin probability for this model has a remarkably simple form if the interoccurrence times are exponentially distributed and also $B^{(1)}$ and $B^{(2)}$ are exponentially distributed (with rates $\mu_{1}$ and $\mu_{2}$, respectively). In particular, in that case

$$
\psi(x)=c_{1} \mathrm{e}^{-\theta_{1} x}+c_{2} \mathrm{e}^{-\theta_{2} x},
$$

where $c_{1}, c_{2} \in \mathbb{R}$ and $\theta_{1}, \theta_{2}$ are positive roots of a certain characteristic equation. This result was proven in [15] with quite some effort by deriving an integro-differential equation for the ruin probability and reformulating it into a delay differential equation of a particular, tractable, type. Cheung et al. [10] later derived this result in a more general setup by conditioning on the distribution of the first undershoot below the initial level and solving the resulting system of integral equations.

In this note we provide a quick alternative probabilistic derivation of (1) for general interoccurrence times (not just exponentially distributed), which can be easily extended to the case of multiple thresholds and phase-type distributed claims.

\section{A short proof of (1) for general interoccurrence times}

The idea is to view this problem as the problem of a continuous first passage downwards for a related spectrally-positive Markov additive process (MAP) [6, Chapter XI] on two states. In fact, only some very basic observations are required to establish that the ruin probability is given by a sum of exponential terms. Some deeper theory is needed to identify the constants.

Let us interchange jumps and interoccurrence times, so that $c A_{k}$ becomes a jump and $B_{k}$ becomes the subsequent interoccurrence time during which the process decreases linearly with slope -1 ; the initial position of this auxiliary process is given by $x+c A_{1}$. Note that the events of ruin for both processes coincide. This auxiliary process $X(t)$ is a MAP with only positive jumps, for which the ruin probability is known. In fact, it is a Markov-modulated linear drift model on two states with jumps at switching times.

Let $J(t) \in\{1,2\}$ denote the state. According to our construction, $J$ stays in state 1 for an $\operatorname{Exp}\left(\mu_{1}\right)$ time, and moves to states 1 or 2 with probabilities $p$ and $1-p$, respectively, where $p=\mathbb{P}\left(A_{1}<a\right)$. Similarly, $J$ stays in state 2 for an $\operatorname{Exp}\left(\mu_{2}\right)$ time, and moves to states 1 or 2 with probabilities $p$ and $1-p$, respectively. The moves of $J$ into state 1 (irrespective of the previous state) cause a jump of $X(t)$ distributed as $c A_{1}$ given $A_{1}<a$, and the moves into state 2 cause a jump of $X(t)$ distributed as $c A_{1}$ given $A_{1} \geq a$ (this is the analogous interpretation to the one in [4] for a risk model with dependence between claims and subsequent interoccurrence times). Let $A^{(1)}$ and $A^{(2)}$ denote random variables distributed as $c A_{1}$ given $A_{1}<a$ and $A_{1} \geq a$, respectively. Finally, in between the moves of the $J$ process, $X$ decreases linearly with slope -1 . For a certain initial distribution of $(X, J)$, to be specified later, we need to determine $\mathbb{P}\left(\tau_{0}^{-}<\infty\right)$, where for $y \geq 0$ we define $\tau_{y}^{-}=\inf \{t \geq 0: X(t)<-y\}$.

Suppose for a moment that $X(0)=0$. Using the memoryless property of the exponential distribution, it is not difficult to see that $J\left(\tau_{y}^{-}\right), y \geq 0$, is also a Markov chain with some transition rate matrix $\boldsymbol{\Lambda}$. It is transient if $X(t)$ (equivalently, the original process) drifts to $\infty$ almost surely (we can also think of adding an additional absorbing state to the state space of 
this Markov chain). Observe that

$$
\mathbb{P}\left(\tau_{y}^{-}<\infty \mid J(0)=1\right)=(1,0) \mathrm{e}^{\Lambda y}\left(\begin{array}{l}
1 \\
1
\end{array}\right) ;
$$

a similar identity is true for $J(0)=2$ (see, e.g. [6, Corollary II.3.5]). The initial distribution of $J$ is given by $(p, 1-p)$, and $X(0)$ is distributed as $x+A^{(i)}$ on $J(0)=i$ for $i=1,2$; hence,

$$
\mathbb{P}_{x}(T<\infty)=p(1,0) \mathbb{E} \mathrm{e}^{\Lambda\left(x+A^{(1)}\right)}\left(\begin{array}{l}
1 \\
1
\end{array}\right)+(1-p)(0,1) \mathbb{E} \mathrm{e}^{\Lambda\left(x+A^{(2)}\right)}\left(\begin{array}{l}
1 \\
1
\end{array}\right)
$$

Remark 1. Replacing the vectors $(1,1)^{\top}$ by $(1,0)^{\top}$ on the right-hand side of (2) would lead to the probability of ruin caused by a claim of type 1 .

It is well known that all the eigenvalues of a transient irreducible transition rate matrix belong to the left half of the complex plane. To see this, we can use Gershgorin's theorem and the fact that irreducibly diagonally dominant matrices are invertible; see, e.g. [13]. Furthermore, these eigenvalues are distinct and real for a $2 \times 2$ matrix, which is easily shown by the examination of the corresponding characteristic equation. Let us denote the eigenvalues of $\boldsymbol{\Lambda}$ by $-\theta_{1}$ and $-\theta_{2}$, where $\theta_{1} \neq \theta_{2}$ and $\theta_{1}, \theta_{2}>0$. Then $\boldsymbol{\Lambda}$ can be written as

$$
\boldsymbol{\Lambda}=\boldsymbol{V}\left(\begin{array}{cc}
-\theta_{1} & 0 \\
0 & -\theta_{2}
\end{array}\right) \boldsymbol{V}^{-1}
$$

where the $2 \times 2$ matrix $\boldsymbol{V}$ is formed from the corresponding eigenvectors. Substituting this into (2) we get the following expression for the ruin probability:

$$
\begin{aligned}
\mathbb{P}_{x}(T<\infty)= & p(1,0) \boldsymbol{V}\left(\begin{array}{cc}
\mathrm{e}^{-\theta_{1} x} G_{1}\left(\theta_{1}\right) & 0 \\
0 & \mathrm{e}^{-\theta_{2} x} G_{1}\left(\theta_{2}\right)
\end{array}\right) \boldsymbol{V}^{-1}\left(\begin{array}{l}
1 \\
1
\end{array}\right) \\
& +(1-p)(0,1) \boldsymbol{V}\left(\begin{array}{cc}
\mathrm{e}^{-\theta_{1} x} G_{2}\left(\theta_{1}\right) & 0 \\
0 & \mathrm{e}^{-\theta_{2} x} G_{2}\left(\theta_{2}\right)
\end{array}\right) \boldsymbol{V}^{-1}\left(\begin{array}{l}
1 \\
1
\end{array}\right),
\end{aligned}
$$

where $G_{i}(\theta)$ is the transform of $A^{(i)}$; e.g. $G_{1}(\theta)=\mathbb{E} \mathrm{e}^{-\theta A^{(1)}}=\mathbb{E}\left[\mathrm{e}^{-\theta c A_{1}} \mid A_{1}<a\right]$. This shows that the ruin probability is of the form (1). It only remains to identify the spectrum of $\boldsymbol{\Lambda}$, i.e. the numbers $\theta_{1}, \theta_{2}$, and the matrix $\boldsymbol{V}$.

\section{Concluding comments}

Identification of the matrix $\boldsymbol{\Lambda}$ is a well-studied problem; see [9] for an iterative procedure and [12] for a spectral method, where the latter also contains a list of earlier works. Firstly, note that there exists a matrix $F(\theta)$ for $\theta \geq 0$, which characterizes our MAP: $\mathbb{E}\left[\mathrm{e}^{-\theta X(t)} ; J(t)=\right.$ $j \mid X(0)=0, J(0)=i]=\left[\mathrm{e}^{F(\theta) t}\right]_{i j}$, with $i, j \in 1,2$. For the present model this matrix is given by

$$
F(\theta)=\left(\begin{array}{cc}
-\mu_{1}+\theta+p \mu_{1} G_{1}(\theta) & (1-p) \mu_{1} G_{2}(\theta) \\
p \mu_{2} G_{1}(\theta) & -\mu_{2}+\theta+(1-p) \mu_{2} G_{2}(\theta)
\end{array}\right) ;
$$

see also [6, Proposition XI.2.2]. Secondly, the spectral method of [12] states that $\theta_{1}$ and $\theta_{2}$ are the two zeros of $\operatorname{det}(F(\theta))$ in the right half of the complex plane, which are positive in our case. Furthermore, the corresponding eigenvectors $\boldsymbol{v}_{i}$, which define $\boldsymbol{V}=\left[\boldsymbol{v}_{1}, \boldsymbol{v}_{2}\right]$, are found from $F\left(\theta_{i}\right) \boldsymbol{v}_{i}=\mathbf{0}$. 
Example 1. Let us consider the particular case when the $A_{k}$ have an exponential distribution of rate $\lambda$. Then

$$
\begin{gathered}
p G_{1}(\theta)=\mathbb{E}\left[\mathrm{e}^{-\theta c A_{1}} ; A_{1}<a\right]=\int_{0}^{a} \lambda \mathrm{e}^{-\lambda t} \mathrm{e}^{-\theta c t} \mathrm{~d} t=\frac{\lambda}{\lambda+\theta c}\left(1-\mathrm{e}^{-(\lambda+\theta c) a}\right), \\
(1-p) G_{2}(\theta)=\mathbb{E}\left[\mathrm{e}^{-\theta c A_{1}} ; A_{1} \geq a\right]=\frac{\lambda}{\lambda+\theta c} \mathrm{e}^{-(\lambda+\theta c) a},
\end{gathered}
$$

which reduces the equation $\operatorname{det}(F(\theta))=0$ to

$$
\left(\theta-\mu_{2}\right)\left(\lambda+\theta c-\mu_{1} c\right)+\lambda\left(\mu_{2}-\mu_{1}\right) \mathrm{e}^{-(\lambda+\theta c) a}=0,
$$

and $\theta_{1}, \theta_{2}$ in (1) are the positive solutions of this equation. This result indeed coincides with the result of [15, Equations (8) and (15)].

Remark 2. The method easily carries over to more general models, similar to those studied in [10]. Firstly, we can have multiple thresholds for $A_{k}$. Then for $m$ intervals the dimension of the matrices $\Lambda$ and $F(\theta)$ will be $m \times m$. Moreover, we can extend the model to claim sizes of phase-type with $\left(n_{i}\right)_{i=1, \ldots, m}$ phases. Then the dimension of the matrices becomes $\left(\sum_{i=1}^{m} n_{i}\right) \times$ $\left(\sum_{i=1}^{m} n_{i}\right)$. In that case the eigenvalues of $\boldsymbol{\Lambda}$ are not necessarily real and distinct. Nevertheless, the spectrum of $\boldsymbol{\Lambda}$ can still be identified; see [12]. The corresponding expression (1) may then include terms of the form $x^{k} \mathrm{e}^{-\theta_{i} x}$ for certain $k$.

Finally, we note that the concept of killing (cf. [14]) can be used in the present context to extend this result on the ruin probability to other ruin-related quantities, e.g. the joint transform of the time to ruin and the deficit at ruin.

\section{References}

[1] Adan, I. J. B. F. And Kulkarni, V. G. (2003). Single-server queue with Markov-dependent inter-arrival and service times. Queueing Systems 45, 113-134.

[2] Ahn, S. And BADEscu, A. L. (2007). On the analysis of the Gerber-Shiu discounted penalty function for risk processes with Markovian arrivals. Insurance Math. Econom. 41, 234-249.

[3] Albrecher, H. And Boxma, O. J. (2004). A ruin model with dependence between claim sizes and claim intervals. Insurance Math. Econom. 35, 245-254.

[4] Albrecher, H. and Boxma, O. J. (2005). On the discounted penalty function in a Markov-dependent risk model. Insurance Math. Econom. 37, 650-672.

[5] Albrecher, H. And Teugels, J. L. (2006). Exponential behavior in the presence of dependence in risk theory. J. Appl. Prob. 43, 257-273.

[6] Asmussen, S. (2003). Applied Probability and Queues, 2nd edn. Springer, New York.

[7] Asmussen, S. and Albrecher, H. (2010). Ruin Probabilities, 2nd edn. World Scientific, Hackensack, NJ.

[8] Boudreault, M., Cossette, H., Landriault, D. and Marceau, E. (2006). On a risk model with dependence between interclaim arrivals and claim sizes. Scand. Actuarial J. 2006, 265-285.

[9] Breuer, L. (2008). First passage times for Markov additive processes with positive jumps of phase type. $J$. Appl. Prob. 45, 779-799.

[10] Cheung, E. C. K., Landriault, D. and Badescu, A. L. (2011). On a generalization of the risk model with Markovian claim arrivals. Stoch. Models 27, 407-430.

[11] Cheung, E. C. K., Landriault, D., Willmot, G. E. and Woo, J.-K. (2010). Structural properties of GerberShiu functions in dependent Sparre Andersen models. Insurance Math. Econom. 46, 117-126.

[12] D’Auria, B., Ivanovs, J., Kella, O. and Mandjes, M. (2010). First passage of a Markov additive process and generalized Jordan chains. J. Appl. Prob. 47, 1048-1057.

[13] Horn, R. A. And Johnson, C. R. (1990). Matrix Analysis. Cambridge University Press.

[14] Ivanovs, J. (2013). A note on killing with applications in risk theory. Insurance Math. Econom. 52, 29-34.

[15] Kwan, I. K. M. And Yang, H. (2007). Ruin probability in a threshold insurance risk model. Belg. Actuarial Bull. 7, 41-49. 